\title{
Folates in Children with Down Syndrome and the Impact of Dietary Supplementation
}

Ryan S. Funk ${ }^{3 *}$, Jordan Jones ${ }^{2}$, Kishore Polireddy ${ }^{3}$, Kanecia O. Zimmerman', Gregory Reed ${ }^{4}$, Nasreen Talib ${ }^{2}$, Mara L. Becker ${ }^{1}$

'Department of Pediatrics, Duke University Hospital, Durham, NC, USA

2Department of Pediatrics, Children's Mercy Kansas City, Kansas City, MO, USA

${ }^{3}$ Department of Pharmacy Practice, University of Kansas, Kansas City, KS, USA

${ }^{4}$ University of Kansas Cancer Center, University of Kansas Medical Center, Kansas City, KS, USA

\section{Article Info}

\section{Article Notes}

Received: May 04, 2020

Accepted: June 09, 2020

\section{${ }^{*}$ Correspondence:}

Ryan S. Funk University of Kansas Medical Center, Department of Pharmacy Practice, 3901 Rainbow Blvd, MS 4047, Rm 6013, Kansas City, KS 66160, USA; Telephone No: 913-945-6904; Fax No: 913-945-2355; Email: ryanfunk@kumc.edu.

${ }^{\circledR} 2020$ Funk RS. This article is distributed under the terms of the Creative Commons Attribution 4.0 International License.

\section{Keywords}

Down Syndrome

Folate

Pediatrics

Dietary Supplement

Vitamins

Precision Medicine

\section{Abstract}

Down syndrome (DS) is the most frequent genetic cause of intellectual disability and recent work has demonstrated that this population has a reduction in erythrocyte (RBC) folate concentrations, which may represent a mechanistic basis for enhanced anti-folate drug toxicity observed in these patients. In this study, folate concentrations were measured in whole blood, plasma, and RBCs in an independent cohort of children with DS ( $n=85)$; and confirms previous findings that this population has lower circulating folate concentrations compared to a control cohort consisting of juvenile arthritis patients $(n=99)$. RBC folate concentrations were $29 \%$ lower in patients with $\mathrm{DS}$, and by multivariable regression analysis reduced RBC folate concentrations were associated with: DS diagnosis $(p=0.0019)$, lack of dietary folate supplementation $(p=0.006)$ and female gender $(p=0.03)$. Patients with DS supplemented with dietary folate in the form of a daily multivitamin (MVI) had $25 \%$ higher RBC folate concentrations ( $p=0.07)$, and were comparable to the $\mathrm{RBC}$ folate concentrations in the control cohort not receiving an MVI. However, girls with DS did not exhibit the same degree of folate repletion as boys who were supplemented with MVI.

These data confirm previous findings that children with DS have reduced circulating folate concentrations; however, those receiving daily MVI had RBC folate concentrations comparable to patients without DS. Female patients with DS appear to have a limited response to folate supplementation compared to males, and thus may be at increased risk for clinical features of folate deficiency and drug toxicity with anti-folate therapies.

\section{Introduction}

Down syndrome (DS), resulting from trisomy of the $21^{\text {st }}$ chromosome, is the most common cause of intellectual disability with an estimated incidence of 14.5 per 10,000 individuals in the US $^{1,2}$. Recognizing that children with DS are at increased risk for the development of toxicities associated with the use of the antifolate drug, methotrexate (MTX), previous efforts in our laboratory have sought to investigate differences in folate homeostasis in this population as the potential mechanistic basis for this clinically observed toxicity ${ }^{3}$. Our recent cross-sectional analysis in a small group of 15 children with DS found that erythrocyte (RBC) folate concentrations were approximately $25 \%$ lower than those in a reference population of children with juvenile arthritis. The objective of this work was to validate these previous findings in an independent cohort of children with DS while accounting for other non-genetic factors impacting folates in this population, including dietary folate supplementation, age, and gender. 
Children with DS are at an increased risk for a number of comorbid autoimmune and oncologic conditions that may require chronic treatment with $\mathrm{MTX}^{4,5}$. However, it is wellrecognized that children with DS have increased sensitivity to the toxic effects of MTX ${ }^{6,7}$. In the treatment of oncologic conditions this has resulted in using lower MTX doses in children with $\mathrm{DS}^{8}$, and although such recommendations don't exist for the treatment of juvenile arthritis, anecdotal evidence supports initiation of MTX at lower doses to reduce the risk of toxicity.

Previous efforts to evaluate the mechanism for enhanced MTX toxicity in children with DS have focused on differences in the plasma pharmacokinetics of MTX but have failed to reproducibly demonstrate any differences ${ }^{9,10}$. However, hyperaccumulation of MTX polyglutamate metabolites have been demonstrated in hyperdiploid acute lymphoblastic leukemia cells containing extra copies of chromosome 21, and have suggested a potential role for enhanced cellular MTX uptake and metabolism in DS that may account for the increase in drug sensitivity ${ }^{11}$. Based on our previous findings of reduced RBC folates in this population ${ }^{3}$, we have hypothesized that a functional depletion of tissue folates would be expected to contribute to the observed increase in MTX toxicity in these patients. Understanding the baseline folate status of patients with DS is a foundational step in investigating this hypothesis, and thus is the basis for this work.

Folates are water soluble vitamins that serve as coenzymes in a variety of one-carbon transfer reactions, including de novo nucleotide biosynthesis and conversion of homocysteine to methionine which is required for various downstream methylation reactions. At the cellular level folate deficiency results in deficits in DNA and RNA biosynthesis, genomic instability, and DNA hypomethylation ${ }^{12}$. Folate deficiency results in a number of clinical features as well, most notably cytopenias (including megoblastic anemia), weakness, fatigue, headache, irritability, as well as gastrointestinal symptoms including nausea, vomiting, and mucosal aphthous ulcerations ${ }^{13}$. These symptoms also represent those commonly observed with MTX toxicity and support the role of a functional folate deficiency in DS that may increase the risk for sensitivity to MTX $^{14,15}$.

A number of enzymes involved in folate transport and metabolism are encoded by genes located on chromosome 21 and provide a potential mechanistic basis for folate dysregulation and enhanced MTX sensitivity in this population. These genes include cystathionine $\beta$-synthase $(C B S)$, phosphoribosylglycinamide formyltransferase (GART), and the reduced folate carrier (RFC or SLC19A1). Increased CBS activity has been hypothesized to result in competition for homocysteine metabolism resulting in folate dysregulation through trapping of 5-methyl- tetrahydrofolate ${ }^{16,17}$. Increased GART activity may result in enhanced folate consumption through de novo purine biosynthesis ${ }^{18}$, resulting in depletion of the intracellular folate pool, and increased RFC activity has been hypothesized to play a role in MTX toxicity as the main transporter responsible for MTX uptake ${ }^{11}$, however the impact of increased RFC expression on systemic folate regulation remains unclear.

In addition to potential genetic causes of metabolic folate dysregulation in children with DS, non-genetic factors such diet, gender, and age must be considered ${ }^{19-21}$. Humans must fully meet their folate needs through their diet since they lack the enzymatic machinery necessary to synthesize their own. As a result, variation in dietary folate intake is a major contributor to interindividual differences in systemic folate status ${ }^{22}$. Therefore, dietary folate supplementation in children with DS may represent an approach to replete tissue folate levels in children with DS as an approach to reduce MTX associated toxicity. However, the impact of folate supplementation on tissue folate stores in children with DS has not been previously evaluated.

In this study we utilized advanced methods to quantitate whole blood, plasma, and RBC folate concentrations in a cohort of children with DS to validate our previous findings of reduced RBC folate concentrations in children with DS. We further evaluate the contribution of folate-containing multivitamin (MVI) use, age, and gender on differences observed in circulating folate concentrations in children with DS.

\section{Materials and Methods}

\section{Patients}

Eighty-five pediatric patients were recruited from the Multidisciplinary Down Syndrome Clinic at Children's Mercy-Kansas City. Patients were enrolled based on convenience of sampling and samples were collected only if clinical laboratory tests were ordered as part of routine care. For each patient enrolled in the study an additional tube of blood was collected for research purposes at the time of routine laboratory testing. Patient data collected included gender, age, use of a folate containing multivitamin (MVI), and hematocrit values measured as part of routine care. Concentrations of folate in whole blood, plasma, and RBCs were measured and compared with those previously reported from a reference population of children with juvenile arthritis (JA) ${ }^{23}$. This study was conducted in accordance with the Code of Ethics of the World Medical Association and was performed under a Children's MercyKansas City Institutional Review Board approved study protocol. Written informed consent was obtained from patient parents or guardians, and patient assent was obtained when appropriate. 


\section{Folate analysis}

Research blood samples collected during phlebotomy for routine clinical laboratory draws were processed immediately following collection. Briefly, aliquots of whole blood were removed prior to separation of plasma from RBCs by centrifugation at $600 \mathrm{xg}$ for 10 minutes. Plasma supernatant was aliquoted, and the RBC pellet was washed thrice in phosphate-buffered saline. The resulting packed RBCs were aliquoted and whole blood, plasma, and RBC samples were stored at $-80{ }^{\circ} \mathrm{C}$ until the time of analysis. Whole blood and plasma concentrations of 5-methyl-tetrahydrofolate (5mTHF) and 5,10-methenyltetrahydrofolate were determined using an adaptation of a previously validated folate analytical method ${ }^{24}$. Briefly, whole blood was thawed on ice and diluted 1:1 in an aqueous deglutamation buffer containing $5 \%$ ascorbic acid and $0.08 \%$ mercaptoethanol and incubated at $37{ }^{\circ} \mathrm{C}$ for 2 hours protected from light. Samples were deproteinated by trichloroacetic acid precipitation and the resulting supernatant was neutralized in phosphate buffer $(\mathrm{pH} 7.0)$ containing $1 \%$ ascorbate and $0.08 \%$ mercaptoethanol. Plasma samples were processed similarly with the exception of the deglutamation step. Following injection samples were separated on an Acquity BEH C18 column $\left(2.1 \times 100 \mathrm{~mm}, 1.7 \mu \mathrm{m}, 40^{\circ} \mathrm{C}\right)$ using a gradient method at flow rate of $0.5 \mathrm{~mL} / \mathrm{min}$ with initial conditions of $98 \%$ mobile phase A $(0.1 \%$ acetic acid $(\mathrm{v} / \mathrm{v})$ in water) and $2 \%$ mobile phase B (25:75 acetonitrile:methanol containing $0.1 \%$ acetic acid $(\mathrm{v} / \mathrm{v})$ ). Following a 0.7 -minute hold mobile phase $\mathrm{B}$ was increased linearly over 2 minutes to $80 \%$, and then returned to $2 \%$ over 0.1 minute with a 1-minute re-equilibration period. Commercially available external and isotope-labelled internal standards were used to prepare a 6-point calibration curve for each analyte. Folate concentrations were determined by interpolation on a standard curve of peak area ratios relative to the isotopelabeled internal standards. RBC folate concentrations were calculated using the method of Lamers et $\mathrm{al}^{25}$ :

$$
R B C \text { folate }=\frac{(\text { whole blood folate } * 100)-(\text { plasma folate } *(100-\text { hematocrit })}{\text { hematocrit }}
$$

\section{Statistical analyses}

Descriptive statistics were performed in the evaluation of the distribution and variability in total folate and folate isoform concentrations in whole blood, plasma, and RBCs. Univariate analyses were performed between clinical variables and RBC folate concentrations using Wilcoxon rank-sum analysis and Spearman's correlation analysis based on the data distribution analysis. Multivariable standard least squares regression analyses was conducted with age, gender, MVI use, and the diagnosis of Down syndrome as explanatory variables for the log transformed total RBC folate concentrations. Statistical analyses were conducted using JMP 11.0 (SAS, Cary, North Carolina).

\section{Results}

For the 85 children with DS recruited for this study, the median [IQR] age was $72[24,138]$ months. Within the DS cohort, 46 (54\%) were female and 29 (34\%) were receiving dietary folate supplementation in the form of a multivitamin (MVI) at the time of sample collection. From the historical cohort of 99 patients with juvenile arthritis that are serving as the reference control population, the median [IQR] age was 151 [104,192] months. In the JA cohort, 60 (61\%) were female and 26 (26\%) were receiving dietary folate supplementation in the form of a daily MVI. The cohorts did not significantly vary by gender or percentage of children receiving dietary folate supplementation, but children in the DS cohort were significantly younger than those in the reference cohort $(\mathrm{p}<0.0001)$.

\section{Circulating folate levels in children with DS}

Folate concentrations were measured in blood samples from our cohort of children with DS and compared to the reference control cohort of children (Figure 1A). Median [IQR] total whole blood folate concentrations in children with DS were found to be $24 \%$ lower compared to the control group $(343[267,453]$ vs $450[300,534] \mathrm{nmol} / \mathrm{L})$. Recognizing that whole blood folate levels represent a composite measure of folate content in both the plasma and circulating blood cells, predominately made up of RBCs, we measured total folate concentrations in the plasma (Figure 1B) and circulating erythrocytes (RBCs) (Figure 1C). In contrast to whole blood folate levels, median [IQR] plasma folate levels were found to be $13 \%$ higher in the children with DS $(48[29,69]$ vs 43 $[27,62] \mathrm{nmol} / \mathrm{L})$. However, this difference was not statistically significant. Median [IQR] RBC folate concentrations in children with DS were found to be $29 \%$ lower compared to the control group (825 [600,1060] vs 1161 [707,1367] nmol/L) and represented the compartment primarily responsible for the lower whole blood folate concentrations observed in this population.

For this analysis, total folate concentrations are comprised of reduced folate isoforms 5-methyltetrahydrofolate (5mTHF) and 5,10-methenyltetrahydrofolate $(5,10-\mathrm{MeTHF})$. The concentrations for each of the folate isoforms were compared between children with DS and the control group (Figure 2). Children with DS were found to have $26 \%$ lower median [IQR] RBC concentrations of 5 mTHF $(767[560,992]$ vs $1033[646,1252] \mathrm{nmol} / \mathrm{L}$, Figure 2A) and 21\% lower RBC concentrations of 5,10-MeTHF $(53[33,78]$ vs $67[36,102]$ nmol/L, Figure 2B) compared to the control group. However, only RBC concentrations of $5 \mathrm{mTHF}$ achieved statistical significance $(\mathrm{p}=0.0021)$. 

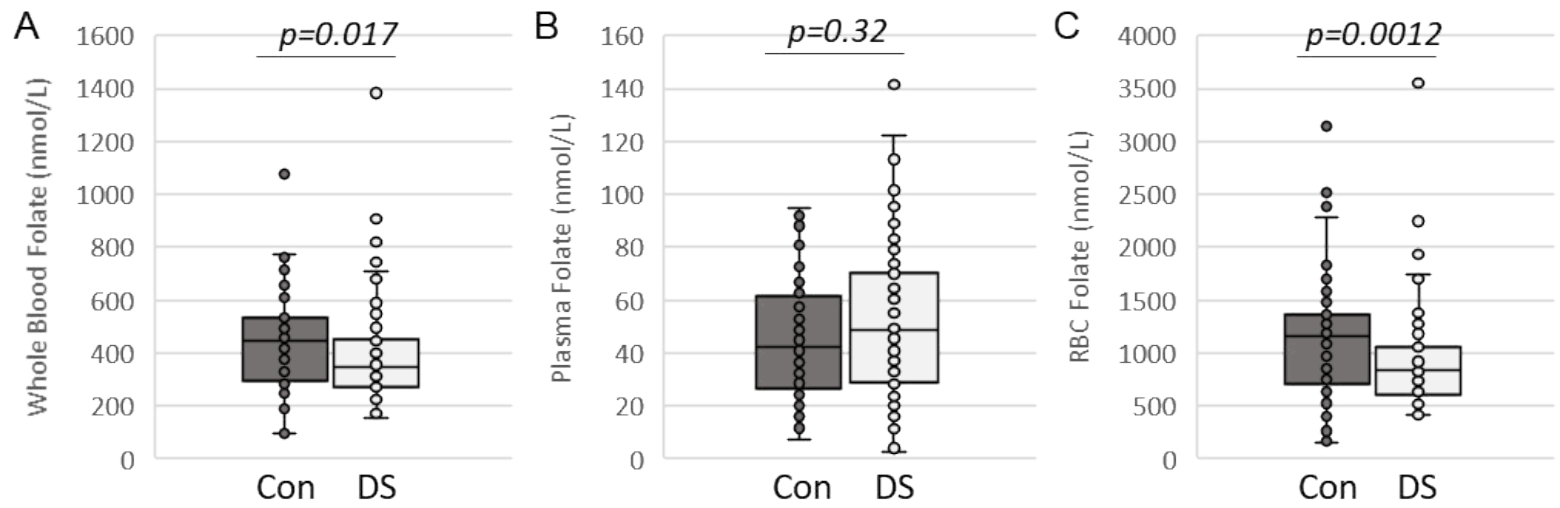

Figure 1: Circulating folate concentrations in children with DS and the reference control group. Total folate concentrations in (A) whole blood, (B) plasma, and (C) RBCs are compared between the reference control group and children with DS. Data are shown as box plots. Lines inside the boxes represent the median. Lines outside the boxes represent the 10th and 90th percentiles. P-values are the result of Wilcoxon rank-sum analysis.

A

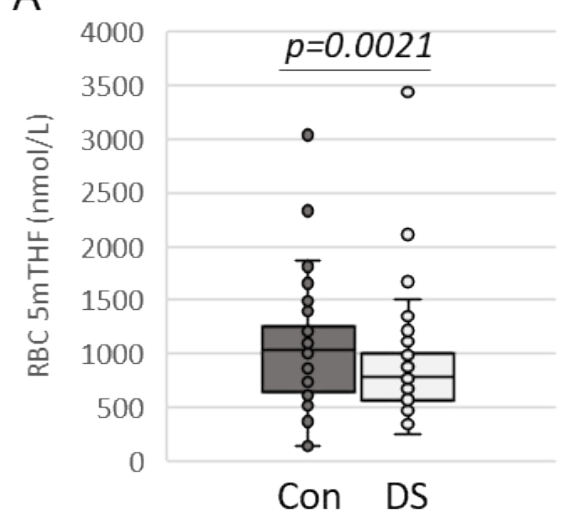

B

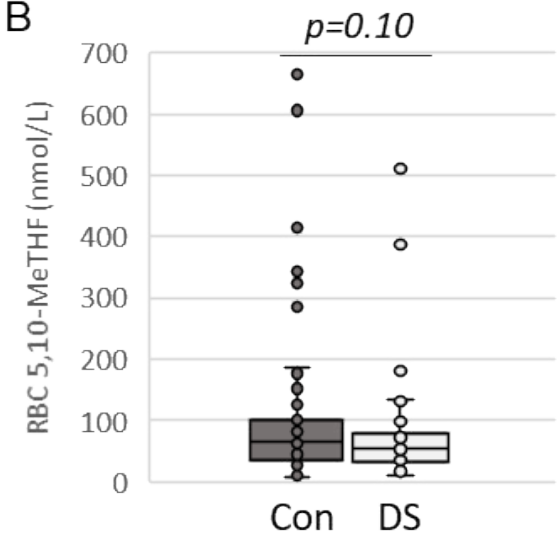

Figure 2: RBC folate concentrations in children with $D S$ and the reference control group. RBC concentrations of $(A)$ $5 \mathrm{mTHF},(\mathrm{B}) 5,10-\mathrm{MeTHF}$ compared between the reference control group and children with DS. Data are shown as box plots. Lines inside the boxes represent the median. Lines outside the boxes represent the 10th and 90th percentiles. P-values are the result of Wilcoxon rank-sum analysis.

Based on previously recognized covariates for folate regulation and the notable demographic differences between the two cohorts, a multivariable linear regression analysis was conducted to assess variables that may contribute to the difference in folate concentrations within the cohorts. The regression model was constructed with log transformed total RBC folate concentrations as the dependent variable and controlling for covariates: age, gender, MVI use, and the diagnosis of DS. The model demonstrated that the diagnosis of DS $(p=0.006)$, lack of MVI use (0.02), and female gender (0.02) are associated with reduced RBC folate concentrations. Age, despite being different between cohorts, did not statistically contribute to the differences in RBC folate in the multivariable model $(\mathrm{p}=0.35)$.

\section{Impact of dietary supplementation on folates in children with DS}

To further interrogate the impact of folate supplementation on circulating folates in children with DS, circulating folate concentrations were stratified based on dietary folate supplementation and compared between the children with DS and the reference control group (Table 1). Children receiving dietary folate in the form of a daily MVI had higher folate concentrations in whole blood, plasma and RBCs, and this was observed for both children with DS and in the control group. However, the percentage difference in whole blood, plasma, and RBC folate concentrations in patients receiving dietary folate supplementation were consistently greater in children with DS. In patients not receiving an MVI, we observed significantly lower whole 


\begin{tabular}{|c|c|c|c|c|}
\hline $\begin{array}{l}\text { Folate, } \\
\text { nmol/L }\end{array}$ & (-)MVI & $(+) M V I$ & $\Delta \%$ & $\mathrm{p}$-value \\
\hline \multicolumn{5}{|l|}{ Control } \\
\hline$n$ & 73 & 26 & & \\
\hline Blood & $\begin{array}{c}414 \\
(279,414)\end{array}$ & $\begin{array}{c}505 \\
(396,619)\end{array}$ & +22 & 0.02 \\
\hline Plasma & $\begin{array}{c}35 \\
(24,52)\end{array}$ & $\begin{array}{c}49 \\
(41,75)\end{array}$ & +40 & 0.002 \\
\hline RBC & $\begin{array}{c}1050 \\
(684,1327)\end{array}$ & $\begin{array}{c}1239 \\
(1067,1539)\end{array}$ & +18 & 0.05 \\
\hline \multicolumn{5}{|c|}{ Down Syndrome } \\
\hline$n$ & 56 & 29 & & \\
\hline Blood & $\begin{array}{c}327 \\
(259,408)\end{array}$ & $\begin{array}{c}444 \\
(284,602)\end{array}$ & +36 & 0.03 \\
\hline Plasma & $\begin{array}{c}42 \\
(24,60)\end{array}$ & $\begin{array}{c}64 \\
(32,76)\end{array}$ & +52 & 0.02 \\
\hline RBC & $\begin{array}{c}772 \\
(596,962)\end{array}$ & $\begin{array}{c}965 \\
(677,1382)\end{array}$ & +25 & 0.07 \\
\hline
\end{tabular}

Table 1. Difference in circulating folate concentrations based on folate containing multivitamin use in the control group and in children with DS. Values presented represent median (IQR) concentrations.

blood ( $\mathrm{p}=0.04)$ and RBC ( $\mathrm{p}=0.004)$ folate concentrations in children with DS compared to the control group. However, among those receiving an MVI, whole blood $(\mathrm{p}=0.18)$ and RBC (0.09) folate concentrations did not statistically significantly differ between children with DS and those in the control group. Moreover, by comparison of median folate concentrations, children with DS receiving an MVI had whole blood ( $p=0.61)$ and RBC $(p=0.92)$ folate levels comparable to the control group of children not receiving an MVI.

Based on the multivariable analysis, in addition to DS and MVI supplementation, female gender was also associated with lower RBC folate concentrations. Therefore, to interrogate the combined contribution of gender and folate supplementation upon folate concentrations, we stratified both cohorts based on gender and folate supplementation and compared whole blood, plasma, and RBC folate concentrations (Table 2). In the control group of patients not receiving MVI, male patients had significantly higher whole blood $(p=0.002)$, and RBC $(p=0.003)$ folate levels compared to females. In the control group cohort receiving MVI, both males and females had measurably higher folate concentrations in each of the matrices (folate concentrations were $43-53 \%$ higher for females on MVI and $26-68 \%$ higher for males on MVI, depending on matrix, Table 2). In contrast, in children with DS not receiving daily MVI, whole blood ( $\mathrm{p}=0.87)$, plasma $(\mathrm{p}=0.80)$, and $\mathrm{RBC}$ $(p=0.89)$ folate concentrations were found to be similar in males and females. Male patients with DS receiving MVI had higher folate concentrations in whole blood, plasma, and RBCs (folate concentrations were $33-67 \%$ higher, depending on matrix, Table 2). However, in females with DS, receipt of MVI was not found to be associated with measurably higher folate concentrations, in fact folate concentrations for female patients on MVI were lower (folate concentrations were $1.5-20 \%$ lower, depending on matrix, Table 2).

\section{Discussion}

Previous work investigating ultrastructural changes in leukocytes in an otherwise healthy population of patients with DS demonstrated an increased risk of macrocytosis associated with reductions in RBC folate ${ }^{26}$. However, studies on folate sufficiency in patients with DS failed to demonstrate a deficiency in serum folates, despite observed macrocytosis ${ }^{27,28}$. This suggests a discrepancy between serum and tissue folate in patients with DS, which is also supported in this work. Specifically, in this study whole blood folate concentrations were found to be significantly lower in children with DS, attributed to reduced RBC folate concentrations (29\% lower), similar to the $25 \%$ lower RBC folate concentrations observed in our previous work. However, despite the markedly lower RBC concentrations, plasma folate concentrations were found to be similar

\begin{tabular}{|c|c|c|c|c|c|c|c|c|}
\hline \multirow{2}{*}{$\begin{array}{l}\text { Folate, } \\
\text { nmol/L }\end{array}$} & \multicolumn{4}{|l|}{ Female } & \multicolumn{4}{|c|}{ Male } \\
\hline & $(-) M V I$ & (+)MVI & $\Delta \%$ & p-value & $(-)$ MVI & (+)MVI & $\Delta \%$ & p-value \\
\hline \multicolumn{9}{|l|}{ Control } \\
\hline $\mathrm{n}$ & 42 & 18 & & & 31 & 8 & & \\
\hline Blood & $\begin{array}{c}319 \\
(250,455) \\
\end{array}$ & $\begin{array}{c}483 \\
(382,529) \\
\end{array}$ & +51 & 0.01 & $\begin{array}{c}481 \\
(391,559) \\
\end{array}$ & $\begin{array}{c}608 \\
(505,748) \\
\end{array}$ & +26 & 0.10 \\
\hline Plasma & $\begin{array}{c}32 \\
(25,58)\end{array}$ & $\begin{array}{c}49 \\
(39,70)\end{array}$ & +53 & 0.03 & $\begin{array}{c}38 \\
(24,49)\end{array}$ & $\begin{array}{c}64 \\
(46,82)\end{array}$ & +68 & 0.02 \\
\hline RBC & $\begin{array}{c}829 \\
(632,1170)\end{array}$ & $\begin{array}{c}1186 \\
(1010,1289) \\
\end{array}$ & +43 & 0.02 & $\begin{array}{c}1232 \\
(1046,1371)\end{array}$ & $\begin{array}{c}1595 \\
(1357,2280)\end{array}$ & +29 & 0.07 \\
\hline \multicolumn{9}{|c|}{ Down Syndrome } \\
\hline$n$ & 32 & 14 & & & 24 & 15 & & \\
\hline Blood & $\begin{array}{c}323 \\
(278,384) \\
\end{array}$ & $\begin{array}{c}318 \\
(208,588) \\
\end{array}$ & -1.5 & 0.88 & $\begin{array}{c}340 \\
(233,441)\end{array}$ & $\begin{array}{c}451 \\
(404,621)\end{array}$ & +33 & 0.01 \\
\hline Plasma & $\begin{array}{c}40 \\
(29,59) \\
\end{array}$ & $\begin{array}{c}32 \\
(18,66)\end{array}$ & -20 & 0.69 & $\begin{array}{c}45 \\
(18,60) \\
\end{array}$ & $\begin{array}{c}75 \\
(58,89) \\
\end{array}$ & +67 & 0.0008 \\
\hline RBC & $\begin{array}{c}776 \\
(612,923)\end{array}$ & $\begin{array}{c}688 \\
(527,1381)\end{array}$ & -11 & 0.46 & $\begin{array}{c}748 \\
(537,1144)\end{array}$ & $\begin{array}{c}1076 \\
(920,1412)\end{array}$ & +44 & 0.01 \\
\hline
\end{tabular}

Table 2. Difference in circulating folate concentrations based on folate containing multivitamin use and gender in the control group and in children with DS. Values presented represent median (IQR) concentrations. 
or slightly higher than those in the reference population. Based on this observation, children with DS appear to have normal plasma/serum folate levels, but a relative deficiency in tissue folates represented by lower RBC folate levels. Therefore, utilizing serum or plasma folate levels as a measure of systemic folate status in children with DS must be approached with caution since these levels may not be representative of tissue folate sufficiency in this population.

As in our previous study, total folate concentrations are represented as the sum of $5 \mathrm{mTHF}$ and $5,10-\mathrm{MeTHF}^{3}$. In our previous work both folate isoforms were found to be significantly lower in RBCs from children with DS, however 5,10-MeTHF was found to be proportionately lower compared to 5mTHF. In this unique cohort of patients with DS, both isoforms were similarly reduced with only RBC 5mTHF reaching statistical significance with median concentrations $24 \%$ lower in the DS population. This finding does not lend support to the hypothesized role of increased CBS activity in children with DS resulting in trapping of $5 \mathrm{mTHF}^{16}$, nor does it support the role of enhanced GARTmediated consumption of 5,10-MeTHF since the relative depletion of the two folate isoforms were similar. It may, however, warrant the utilization of more specific reduced and methylated forms of folate (such as folinic acid or 5mTHF) for supplementation when MTX toxicity is observed, as these reduced forms have also shown a greater rescue effect upon cell proliferation in the presence of MTX in fibroblasts from patients with $\mathrm{DS}^{29}$. This observed folate deficiency in patients with DS is particularly intriguing when taking into account reported gene-environment risk factors in certain populations that support the notion that peri-conceptional nutritional supplementation may reduce the risk of having a child with DS in mothers carrying polymorphisms in certain folate pathway genes ${ }^{30}$. These findings of folate depletion in children with DS also highlight recent work describing the impact of folate depletion on brain structure and function in a mouse model of DS with the possible implication that folate insufficiency promotes the progression of structural and functional abnormalities in $\mathrm{DS}^{31}$. However, further research is needed to investigate the role of folate insufficiency in cognitive dysfunction in DS and the potential role for folate status monitoring and supplementation as an approach to prevent cognitive decline.

Stratification of our study cohorts based on MVI use demonstrated that folate supplementation in the DS population was associated with proportionately higher RBC folate concentrations. In fact, MVI use in the DS population as a whole resulted in $\mathrm{RBC}$ folate concentrations comparable to the non-DS cohort with or without supplementation. This data supports routine folate supplementation with an MVI in children with DS is an effective means to replete tissue folate. However, our data suggests that response to folate supplementation may be sex dependent.

In contrast to the control population not receiving folate supplementation where male patients had increased RBC folate concentrations compared to females; patients with DS not receiving folate supplementation had similar RBC folate concentrations regardless of sex. Moreover, in the control population, patients receiving folate supplementation had measurably higher RBC folate concentrations in both males and females. Alternatively, in the DS population receiving folate supplementation, only male patients had measurably higher RBC folate concentrations, and no increase in RBC concentrations were observed in supplemented female DS patients (in fact the concentrations were lower in supplemented female patients). The lack of folate response with supplementation in females was not only observed in RBCs but was also in plasma, which tends to be more responsive to dietary folate supplementation. This observation was surprising based on previous studies demonstrating that females tend to be more responsive to dietary folate supplementation than males ${ }^{32}$.

This study has limitations as a cross sectional study utilizing convenience sampling. It is possible that this study is underpowered to detect gender differences in response to MVI, or this observation may be an artifact of the cross-sectional observational nature of the study. However, we would expect to see similar trends in females in the control cohort as well if these potential limitations were in fact valid. It is possible there was selective recall bias when collecting medication histories in the patients with DS, although processes for medication reconciliation are consistent across all patients in the DS clinic, thus we would not expect there to be a selective recall bias in female patients only. All DS samples were processed at the same time via the same methods.

If these findings were confirmed it could suggest that females with DS may have a deficiency in the absorption or conversion of dietary folic acid to its bioactive form. This could mean that females require an increase in folic acid dose or may possibly require supplementation with a bioactive form of folate, such as leucovorin or $5 \mathrm{mTHF}$. Sexspecific differences in enzyme transporter function in DS is currently unknown. However, sex specific differences in blood brain barrier transporters in rats (such as Oatp1a4) has been described ${ }^{33}$, and differential enzyme expression in males and females for several enzymes within the folate pathway, thought to be influenced by different proportions of sex hormones upon these enzymes, has also been reported ${ }^{34}$. However, our observation was specific to female children affected also with DS, thus any hypothesized impact on transporter activity would need to be specific to the DS population and related in some way to trisomy of the $21^{\text {st }}$ chromosome. Thus, the suggestion that females 
with DS need an alternative strategy of dietary folate supplementation must first be replicated and validated through a prospective dietary folate supplementation study.

Together, this work confirms our previous work demonstrating reduced $\mathrm{RBC}$ folate concentrations in children with DS. Furthermore, it demonstrates that folate supplementation in the form of an MVI is associated with increased RBC folate concentrations similar to those observed in a non-DS pediatric population. However, the response to MVI appeared to be specific to males, and suggests that females with DS may not be as responsive to routine folate supplementation, however, this requires further investigation.

\section{Acknowledgements}

The authors would like to thank Chelsey Smith for assistance in patient recruitment and sample collection for this study.

\section{Financial Support}

Support for this project was provided by a Patton Trust Grant awarded by the Kansas City Area Life Sciences Institute, Young Investigator Award, awarded by Children's Mercy Hospital, Kansas City, a Research Starter Grant from the PhRMA Foundation in Pharmacology/Toxicology, the Down Syndrome Guild of Greater Kansas City, a CTSA grant from the National Center for Advancing Translational Sciences awarded to the University of Kansas for Frontiers: University of Kansas Clinical and Translational Science Institute (\#KL2TR002367), and a Centers for Biomedical Research Excellence grant from the National Institute for General Medical Sciences to the University of Kansas Medical Center in support of the Kansas Institute for Precision Medicine (\#P20GM1304230).

\section{References}

1. Parker SE, Mai CT, Canfield MA, et al. Updated National Birth Prevalence estimates for selected birth defects in the United States, 2004-2006. Birth Defects Res A Clin Mol Teratol. 2010; 88(12): 10081016.

2. Pritchard M, Reeves RH, Dierssen M, et al. Down syndrome and the genes of human chromosome 21: current knowledge and future potentials. Report on the Expert workshop on the biology of chromosome 21 genes: towards gene-phenotype correlations in Down syndrome. Washington D.C., September 28-October 1, 2007. Cytogenet Genome Res. 2008; 121(1): 67-77.

3. Funk RS, Talib NJ, Zimmerman KO, et al. Altered Folate Homeostasis in Children with Down Syndrome: A Potential Basis for Enhanced Methotrexate Toxicity. J Pediatr. 2020.

4. Baum RA, Nash PL, Foster JE, et al. Primary care of children and adolescents with down syndrome: an update. Curr Probl Pediatr Adolesc Health Care. 2008; 38(8): 241-261.

5. Padmakumar B, Evans Jones LG, Sills JA. Is arthritis more common in children with Down syndrome? Rheumatology (Oxford). 2002; 41(10): 1191-1193.
6. Jones JT, Talib N, Lovell D, et al. Clinical Features and Treatment of Down Syndrome Arthropathy: Experience from Two US Tertiary Hospitals. Paediatr Drugs. 2019; 21(1): 33-39.

7. Peeters M, Poon A. Down syndrome and leukemia: unusual clinical aspects and unexpected methotrexate sensitivity. Eur J Pediatr. 1987; 146(4): 416-422.

8. Kroll M, Kaupat-Bleckmann K, Morickel A, et al. Methotrexateassociated toxicity in children with Down syndrome and acute lymphoblastic leukemia during consolidation therapy with high dose methotrexate according to ALL-BFM treatment regimen. Haematologica. 2020; 105(4): 1013-1020.

9. Buitenkamp TD, Mathot RA, de Haas V, et al. Methotrexate-induced side effects are not due to differences in pharmacokinetics in children with Down syndrome and acute lymphoblastic leukemia. Haematologica. 2010; 95(7): 1106-1113.

10. Garre ML, Relling MV, Kalwinsky D, et al. Pharmacokinetics and toxicity of methotrexate in children with Down syndrome and acute lymphocytic leukemia. J Pediatr. 1987; 111(4): 606-612.

11. Taub JW, Ge Y. Down syndrome, drug metabolism and chromosome 21. Pediatr Blood Cancer. 2005; 44(1): 33-39.

12. Pietrzik K. Folate deficiency: morphological and functional consequences. Bibl Nutr Dieta. 1989; (44): 123-130.

13. Khan KM, Jialal I. Folic Acid (Folate) Deficiency. In: StatPearls. Treasure Island (FL). 2020.

14. Solomon DH, Glynn RJ, Karlson EW, et al. Adverse Effects of Low-Dose Methotrexate: A Randomized Trial. Ann Intern Med. 2020.

15. Moe PJ, Holen A. High-dose methotrexate in childhood all. Pediatr Hematol Oncol. 2000; 17(8): 615-622.

16. Ueland PM, Refsum H, Christensen B. Methotrexate sensitivity in Down's syndrome: a hypothesis. Cancer Chemother Pharmacol. 1990; 25(5): 384-386.

17. Pogribna M, Melnyk S, Pogribny I, et al. Homocysteine metabolism in children with Down syndrome: in vitro modulation. Am J Hum Genet. 2001; 69(1): 88-95.

18. Knox AJ, Graham C, Bleskan J, et al. Mutations in the Chinese hamster ovary cell GART gene of de novo purine synthesis. Gene. 2009; 429(12): 23-30.

19. Dietrich M, Brown CJ, Block G. The effect of folate fortification of cereal-grain products on blood folate status, dietary folate intake, and dietary folate sources among adult non-supplement users in the United States. J Am Coll Nutr. 2005; 24(4): 266-274.

20. Papandreou D, Mavromichalis I, Makedou A, et al. Total serum homocysteine, folate and vitamin B12 in a Greek school age population. Clin Nutr. 2006;25(5):797-802.

21. Kerr MA, Livingstone B, Bates CJ, et al. Folate, related B vitamins, and homocysteine in childhood and adolescence: potential implications for disease risk in later life. Pediatrics. 2009; 123(2): 627-635.

22. Pfeiffer CM, Hughes JP, Lacher DA, et al. Estimation of trends in serum and RBC folate in the US. population from pre- to postfortification using assay-adjusted data from the NHANES 1988-2010. J Nutr. 2012; 142(5): 886-893.

23. Becker ML, van Haandel L, Gaedigk R, et al. Red blood cell folate concentrations and polyglutamate distribution in juvenile arthritis: predictors of folate variability. Pharmacogenet Genomics. 2012; 22(4): 236-246.

24. van Haandel L, Becker ML, Williams TD, et al. Comprehensive quantitative measurement of folate polyglutamates in human erythrocytes byion pairing ultra-performanceliquid chromatography/ tandem mass spectrometry. Rapid Commun Mass Spectrom. 2012; 26(14): 1617-1630. 
25. Lamers Y, Prinz-Langenohl R, Bramswig S, et al. Red blood cell folate concentrations increase more after supplementation with [6S]-5methyltetrahydrofolate than with folic acid in women of childbearing age. Am J Clin Nutr. 2006; 84(1): 156-161.

26. Gericke GS, Hesseling PB, Brink $S$, et al. Leucocyte ultrastructure and folate metabolism in Down's syndrome. S Afr Med J. 1977; 51(12): 369-374.

27. Akin K. Macrocytosis and leukopenia in Down's syndrome. JAMA. 1988; 259(6): 842.

28. David O, Fiorucci GC, Tosi MT, et al. Hematological studies in children with Down syndrome. Pediatr Hematol Oncol. 1996; 13(3): 271-275.

29. Vitale L, Serpieri V, Lauriola M, et al. Human trisomy 21 fibroblasts rescue methotrexate toxic effect after treatment with 5-methyltetrahydrofolate and 5-formyl-tetrahydrofolate. J Cell Physiol. 2019.
30. Sukla KK, Jaiswal SK, Rai AK, et al. Role of folate-homocysteine pathway gene polymorphisms and nutritional cofactors in Down syndrome: A triad study. Hum Reprod. 2015; 30(8): 1982-1993.

31. Helm S, Blayney M, Whited T, et al. Deleterious Effects of Chronic Folate Deficiency in the Ts65Dn Mouse Model of Down Syndrome. Front Cell Neurosci. 2017; 11: 161.

32. Winkels RM, Brouwer IA, Verhoef $\mathrm{P}$, et al. Gender and body size affect the response of erythrocyte folate to folic acid treatment. J Nutr. 2008; 138(8): 1456-1461.

33. Brzica H, Abdullahi W, Reilly BG, et al. Sex-specific differences in organic anion transporting polypeptide 1a4 (Oatp1a4) functional expression at the blood-brain barrier in Sprague-Dawley rats. Fluids Barriers CNS. 2018; 15(1): 25.

34. Sadre-Marandi F, Dahdoul T, Reed MC, et al. Sex differences in hepatic one-carbon metabolism. BMC Syst Biol. 2018; 12(1): 89. 